\title{
On the Propagation of Waves in an Anisotropic Solid with Laser-Induced Atomic Defects
}

\author{
F. Kh. Mirzade \\ Institute on Laser and Information Technologies, Russian Academy of Sciences, Moscow 140700, Russia \\ Correspondence should be addressed to F. Kh. Mirzade; fmirzade@rambler.ru
}

Received 16 October 2014; Accepted 23 December 2014

Academic Editor: Sergei Sergeenkov

Copyright (C) 2015 F. Kh. Mirzade. This is an open access article distributed under the Creative Commons Attribution License, which permits unrestricted use, distribution, and reproduction in any medium, provided the original work is properly cited.

\begin{abstract}
The behavior of plane waves in a linear, elastic anisotropic laser-excited solid has been investigated taking into account the effects of atomic defect generation. It is found that there are four types of dispersive waves in these crystals, namely, a quasilongitudinal (QLmode), two quasitransverse (QT-mode), and a quasidefect concentration (N-mode) wave. The complex secular equations for cubic and transversely isotropic crystals are reduced as special cases. It is demonstrated that when waves propagate in one of the planes of transversely isotropic solid having defect concentration field, only one purely quasitransverse wave decouples from the rest of the motion and is not influenced by defect concentration changes. The other waves are coupled and get modified due to presence of defects. When waves propagate along the axis of the solid, only QT- and N-mode are coupled, whereas the two QT-modes get decoupled from the rest of the motion. The phase velocities and attenuation factors of waves have been obtained. Significant effect of defects and anisotropy on wave characteristics is observed in certain ranges of frequency. It is also shown that there is an appreciable variation in case of QL-mode as compared with QT- and N-mode.
\end{abstract}

\section{Introduction}

The study of dynamic properties and mechanical behaviors of elastic solids is significant in the ultrasonic inspection of materials, vibrations of structures, micro- and nanotechnologies, and various other fields. Such materials are usually described by equations of linear elasticity. However, there are materials of a more complex microstructure: laser-excited solids with atomic defect (vacancy, interstitial atom) generation, granular materials, materials with nanovoids, and so forth. Here the modeling of physical processes requires an approach, based, in general, on the use of the internal energy balance, linear momentum balance, and concentration balance equations.

The mechanical waves (lattice straining) propagating in a condensed medium carry information about distortions of their form and energy and about the energy losses related to the defect structure; this piece of information is needed for optical-acoustical diagnostics of various parameters and the structure of solids. Also the investigation of generation and diffusion effects in defect's subsystem on elastic wave propagation can play an important role in understanding many laser-induced processes in the solids, particularly in the laser fast recrystallization, laser annealing, selective laser sintering of powders, multipulse laser etching, and pulsed laser-assisted thin-film deposition.

During the last two decades [1-4], the classical theory of elasticity has been extended to laser-excited solids with atomic defect generation to describe the instabilities and selforganization of various strain-diffusive periodic structures (micro- and nanosized) on the surfaces and the volume. This theory defect density includes an additional independent kinematic variable and reduces to classical elasticity when the defect generation is absent. The defect concentration field, in above studies, has been described by the kinetic equation in the diffusion-drift approximation, including the nonlinear strain-induced generation/annihilation processes in defect's subsystem. The strain-induced diffusion of defects in elastic solids is due to coupling of the fields of defect concentration and strain of the medium through the deformation potential of defects. The 1D and 2D self-organization of nonlinear coupled localized strain-defect structures (solitons or solitary 
waves) due to concentration-elastic instability were also considered [5-8]. Mathematical models of these studies were based on coupled nonlinear equations for the displacement and atomic defect concentration fields. The theory of mechanical waves coupled to atomic defect dynamics and including thermal change effects in solids subjected to pulsed laser irradiation has been considered by Mirzade [9] and Bargmann and Favata [10]. Some features of the physical problems coupling diffusion, mechanics, and thermal waves in a geometrically nonlinear solid have been studied in [11, 12].

An overview of methods for analysis of elastic fields in solids from various structural defects (dislocation, inhomogeneous inclusions, grain boundaries, and cracks) was presented by Mura [13] using the eigenstrain concept of Eshelby in micromechanics. In Mura's eigenstrain theory, the eigenstrains corresponding to each defect are conveniently expressed in terms of a defect concentration. This theory has been applied successfully to modeling of many important processes in crystalline solids such as diffusional phase transformations and microstructure coarsening, which involve diffusional redistribution of atoms under the influence of stresses arising from coherent compositional inhomogeneities as well as from structural defects $[14,15]$. The spatial distribution of defects in these models is described by the space-dependent eigenstrains. New perspectives on the phase field approach in modeling deformation and material defects as well as microstructural evolution (grain growth, precipitate evolution, and solute segregation) are reviewed in [16]. Most of the studies on waves coupled to defect dynamics in elastic media discuss the propagation in isotropic media.

Investigations of waves in anisotropic materials are considerably more difficult than the classical and well-understood isotropic problem. The theory of elastic wave behavior propagation in anisotropic solids is well known [17, 18]. Extensive theoretical efforts have been made so far to model propagation of plane harmonic waves in heat conducting anisotropic elastic solids [19-24]. The study of wave propagation in generalized thermoelastic anisotropic media with additional parameters like prestress, porosity, thermoelastic diffusion, viscosity, microstructure, and other parameters allowed us to obtain vital information about existence of new or modified waves. The eigenvalue problems of elastic waves in piezoelectric anisotropic solids were studied by Guo [25]. Valuable attempts have been carried out in [26] to investigate the propagation of waves in a homogeneous, transversely isotropic, piezothermoelastic plate. Acharya et al. investigated the general theory of transversely isotropic magnetoelastic interface waves in conducting media under initial hydrostatic tension or compression [27].

Keeping in view the increased usage of anisotropic materials in the laser additive micro- and nanotechnologies, the aim of the present investigation is to study the propagation characteristics of bulk mechanical waves in infinitely extended anisotropic laser-excited solids with defect generation in the context of coupled concentration-elasticity theory. It is a continuation of our previous works $[1,2]$. In general, the secular equation obtained yields four roots. These roots can be associated with four waves, namely, a quasi-longitudinal (QL) wave, two quasi-transverse (QT) waves, and a quasidefect concentration wave (N-mode). All waves are found to attenuate in various directions of propagation and to be dispersive. Significant effect of defects, relaxation time, and anisotropy on wave characteristics is observed in certain ranges of frequency. The general characteristics equation for the crystals with transversely isotropic and cubic symmetry has been solved by using series (perturbation) expansion methods in order to obtain phase velocity and attenuation coefficient of the waves. Dispersion curves and graphs of attenuations are given for the single crystal of zinc ( $\mathrm{Zn}$ ) depending on various direction of propagation. Some particular cases of interest are also considered.

\section{Formulation of the Problem}

Consider an unbounded, linear anisotropic elastic solid with mobile atomic defects (vacancy, interstitial atom) generated by external energy fluxes (e.g., laser pulses). We use a fixed Cartesian coordinate system $x_{i}, i=1,2,3$. Let $u_{i}$ be the components of displacement vector and $N$ the lattice defect concentration. There could be two types of defects (vacancies and interstitial atoms) but we limit our consideration to one (for definiteness, interstitial atoms). The dynamical model that can describe the evolution of such a system should be based on (i) the evolution of atomic defect concentration in a strained solid and (ii) the strain field of a solid in the presence of a nonuniform defect concentration field.

The stress-strain constitutive equation is given by [28]

$$
\sigma_{i k}=c_{i k j m}\left(e_{j m}-e_{j m}^{d}\right)
$$

Here $\sigma_{i k}\left(=\sigma_{k i}\right)$ and $e_{i k}\left(=e_{k i}\right)$ are the components of the stress and strain tensors, respectively; $c_{i k j m}, i, k, j, m=1,2,3$, are the components of the fourth-order elasticity tensor. $e_{j m}^{d}=$ $\Omega_{j m} N$ is the strain (or eigenstrain) of the medium due to difference between the covalent radii of the lattice atoms and point defects. The dilatation tensor $\Omega_{j m}$ characterizes the lattice deformation due to the appearance of a single point defect in the crystal [28]. Strain-displacement relation is as follows: $2 e_{j m}=\nabla_{m} u_{j}+\nabla_{j} u_{m}$. We note that (1) has the same structure as the constitutive relation for thermoelasticity with $N$ and $\Omega_{j m}$ playing the role of temperature and thermal expansion coefficient, respectively.

In this paper, we will consider the problem of the wave propagation in an anisotropic solid irradiated over a large area by CW or pulsed lasers. Furthermore, we will assume that the temperature field of the medium has reached its equilibrium value. Its evolution is sufficiently slow compared to atomic defect generation and can be considered quasistationary. We also assume that the contribution of thermal strains to deformation fields is negligible compared to lattice dilatation due to atomic defects and the phase changes and chemical reactions in the medium are absent. 
The basic governing equations for the displacement and defect concentration fields for the case of an anisotropic medium in the absence of body forces can be written as

$$
\begin{gathered}
\rho \frac{\partial^{2} u_{i}}{\partial t^{2}}=c_{i k j m} \nabla_{k} \nabla_{m} u_{j}-\vartheta_{i k} \nabla_{k} N \\
\frac{\partial N}{\partial t}-g+r N=D_{i j} \nabla_{i} \nabla_{j} N-\nabla_{i}\left(N v_{i}\right) .
\end{gathered}
$$

In the above equations, $\rho$ is the density of the medium; $D_{i j}$ is the tensor of diffusion coefficient of the defects; the deformational tensor $\vartheta_{i k}$ controls the strain-defect interaction $\left(\vartheta_{i k}=\right.$ $\left.c_{i k j m} \Omega_{j m}\right) ; g$ and $r$ are the thermal-fluctuation generation rate of atomic defects at sites and the recombination rate of defects on microstructural sinks, respectively.

Strain field affects the characteristics of the atomic defects. Thus, when the strain waves propagate, the formation energy of defects changes in the compression and dilatation zones. If $\vartheta_{i k}^{g}$ is the deformation potential characterizing the variation of the formation activation energy of defects under the lattice deformation, the renormalized formation energy of atomic defects can be represented as $w_{g}=w_{g 0}-\vartheta_{i k}^{g} e_{i k}\left(w_{g 0}\right.$ is the formation energy for defects in an unstrained crystal). If there is a deformation-related perturbation of the lattice, not only does the formation energy of defects decrease, but also the activation energy for the defect migration decreases $w_{m}=w_{m 0}-\vartheta_{i k}^{m} e_{i k}\left(w_{m 0}\right.$ is the migration energy of the defects in the absence of deformation and $\vartheta_{i k}^{m}$ is the deformation potential characterizing the variation of the migration activation energy of defects under the lattice deformation); this results in an increase in the diffusion coefficient of defects. Then for the generation $(g)$ and recombination $(r)$ rates of atomic defects we can write the following expressions:

$$
\begin{aligned}
& g=g_{0} \exp \left(-\beta \vartheta_{i k}^{g} e_{i k}\right), \\
& r=r_{0} \exp \left(-\beta \vartheta_{i k}^{m} e_{i k}\right) .
\end{aligned}
$$

Here $g_{0}=g_{0}^{\prime} \exp \left(-\beta w_{g 0}\right)$ is the coefficient representing the thermal-fluctuation generation of atomic defects at sites in unstrained crystal $\left(\beta^{-1}=k_{B} T, T\right.$ is the absolute temperature, $k_{B}$ is the Boltzmann constant, and $g_{0}^{\prime}$ is the constant); $r_{0}^{-1}=$ $\tau^{\prime} \exp \left(w_{m 0} / k_{B} T\right)$ is the relaxation time of defects in the absence of the strain field ( $\tau^{\prime}$ is the relaxation rate constant).

The components of the defect drift velocity $v_{i}$ induced by strain-defect interaction are

$$
v_{i}=D_{i k} \beta F_{k}=-D_{i k} \beta \nabla_{k} U^{\text {int }}=\beta D_{i k} \vartheta_{j l} \nabla_{k} e_{j l}
$$

We assume that the above constitutive coefficients and the diffusivity tensor satisfy the symmetry relations: $c_{i k j m}=$ $c_{k i j m}=c_{i k m j}=c_{j m i k}, \vartheta_{i k}=\vartheta_{k i}$, and $D_{i k}=D_{k i}$.

Parameters in (1)-(3) are assumed to satisfy the following conditions: (i) the diffusivity tensor $D_{i j}$ is symmetric and positive-definite, (ii) the elastodefect coupling constants are nonsingular, (iii) the linear elasticities are positive-definite $\left(c_{i k l m} e_{i j} e_{k l}>0\right)$.
We can express the defect concentration field as $N=$ $N_{0}+N^{*}\left(N_{0}=g_{0} \tau\right.$ is a spatially homogeneous solution; $N^{*}$ is small nonhomogeneous perturbations). Inserting in (3) and neglecting the nonlinear terms, we get the linearized equation as

$$
\begin{aligned}
\frac{\partial N^{*}}{\partial t} & +\tau^{-1} N^{*}-D_{i j} \nabla_{i} \nabla_{j} N^{*} \\
& =g_{0} \vartheta_{i k}^{(g m)} e_{i k}-\beta N_{0} \nabla_{i}\left(D_{i k} \vartheta_{j l} \nabla_{k} e_{j l}\right),
\end{aligned}
$$

where $\vartheta_{i k}^{(g m)}=\vartheta_{i k}^{(g)}-\vartheta_{i k}^{(m)}$ and $\tau=r_{0}^{-1}$.

\section{Secular Equation for the Plan Waves in Inbounded Medium}

For plane harmonic waves, we assume the solution of the form

$$
\left(u_{k}, N^{*}\right)=\left(\bar{u}_{k}, \bar{N}\right) \exp \left[i\left(\xi x_{p} n_{p}-\omega t\right)\right]
$$

where $\omega$ is the circular frequency and $\xi$ is the wave number; $\left(\bar{u}_{k}, \bar{N}\right)$ are the undetermined amplitude vectors that are independent of time $(t)$ and coordinates $\left(x_{p}\right) \cdot n_{p}(p=1,2,3)$ are the components of the unit wave normal $(\vec{n})$ giving the propagation direction.

Substitution of (7) into (1), (2), and (6) yields

$$
\begin{gathered}
\left(\gamma_{i k} \xi^{2}-\rho \delta_{i k} \omega^{2}\right) \bar{u}_{k}-i \xi \vartheta_{i j} n_{j} \bar{N}=0, \\
\left(g_{0} \vartheta_{i k}^{(g m)} n_{k}+\xi^{2} D N_{0} \vartheta_{l k} n_{k}\right) i \xi \beta \tau \bar{u}_{l}+\left(\xi^{2} l^{2}-i \omega \tau+1\right) \bar{N}=0 .
\end{gathered}
$$

Here $l=\sqrt{D \tau}$ and $\delta_{i k}$ is the Kronecker delta function. Christoffel's tensor may be expressed as follows:

$$
\gamma_{i k}=c_{i j k m} n_{m} n_{j}, \quad D=D_{i j} n_{i} n_{j} .
$$

The system of (8) has a nontrivial solution for $\bar{u}_{k}$ and $\bar{N}$ if the determinant of their coefficients vanishes. This leads to

$$
l^{2} \xi^{2} \operatorname{det}\left[\xi^{2} \gamma_{i k}-\rho \omega^{2} \delta_{i k}\right]-(i \omega \tau-1) \operatorname{det}\left[\xi^{2} \tilde{\gamma}_{i k}-\rho \omega^{2} \delta_{i k}\right]=0 \text {, }
$$

where

$$
\begin{aligned}
& \tilde{\gamma}_{i k}=\gamma_{i k}+\frac{\beta \tau \vartheta_{k j} n_{j}\left(g_{0} \vartheta_{i l}^{(g m)} n_{l}+N_{0} D \xi^{2} \vartheta_{i l} n_{l}\right)}{(i \omega \tau-1)}, \\
& \operatorname{det}\left[\xi^{2} \Gamma_{i k}-\delta_{i k} \rho \omega^{2}\right] \\
& =\left|\begin{array}{ccc}
\xi^{2} \Gamma_{11}-\rho \omega^{2} & \xi^{2} \Gamma_{12} & \xi^{2} \Gamma_{13} \\
\xi^{2} \Gamma_{21} & \xi^{2} \Gamma_{22}-\rho \omega^{2} & \xi^{2} \Gamma_{23} \\
\xi^{2} \Gamma_{31} & \xi^{2} \Gamma_{32} & \xi^{2} \Gamma_{33}-\rho \omega^{2}
\end{array}\right| .
\end{aligned}
$$

In (12) $\Gamma_{i k}=\gamma_{i k}$ or $\tilde{\gamma}_{i k}$ 
Equation (10) is the dispersion equation for plane harmonic waves and contains all information regarding the phase velocities and attenuation (amplification) coefficient of the waves.

On account of properties (i)-(iii) and the symmetric character of stress and strain tensors, the tensors $\gamma_{i k}$ and $\widetilde{\gamma}_{i k}$ are symmetric and positive-definite. Since the tensor $\vartheta_{i k}$ is nonsingular, the scalar $\alpha=\gamma_{i k} \vartheta_{i p} \vartheta_{k q} n_{p} n_{q} / \vartheta_{r j} \vartheta_{r s} n_{j} n_{s}$ is positive, which can be used for purposes of nondimensionalization.

We make the frequency $(\omega)$ to be real and the wavenumber $(\xi)$ as complex. Taking $\xi=k+i q$ ( $k, q$ real), the exponent in the plane wave solution (7) becomes $\exp \left(-q x_{p} n_{p}\right) \exp \left\lfloor i k\left(x_{p} n_{p}-v t\right)\right\rfloor$. This shows that $v=\omega / k$ is the propagation speed and $q$ is the attenuation coefficient of the waves. For $x_{p} n_{p}>0$, if $q<0$, the wave amplitudes are amplified, while for $q>0$ the wave attenuates in space. in $\xi$ as

Equation (10) yields the following characteristic equation

$$
\begin{aligned}
& \sigma \alpha^{4} a_{3} \xi^{8}-\rho \omega^{2} \alpha^{3}\left(\sigma a_{2}+A_{3} \tau_{0}\right) \xi^{6} \\
& \quad+\rho^{2} \omega^{4} \alpha^{2}\left(\sigma a_{1}+A_{2} \tau_{0}\right) \xi^{4}-\rho^{3} \omega^{6} \alpha\left(\sigma+A_{1} \tau_{0}\right) \xi^{2} \\
& \quad+\tau_{0} \rho^{4} \omega^{8}=0
\end{aligned}
$$

where $\tau_{0}=i \sigma / \chi-1, \sigma=\chi^{2} \omega^{*} \tau, \chi=\omega / \omega^{*}, \omega^{*}=\alpha / \rho D$, and $a_{i}$ and $A_{i}(i=1,2,3)$ are given by the following expressions:

$$
\begin{gathered}
a_{1}=b_{11}+b_{22}+b_{33}, \\
a_{2}=b_{11} b_{22}+b_{22} b_{33}+b_{11} b_{33}-b_{12} b_{21} \\
-b_{23} b_{32}-b_{13} b_{31}, \\
a_{3}=b_{11} b_{22} b_{33}+b_{12} b_{23} b_{31}+b_{13} b_{21} b_{32} \\
-b_{11} b_{23} b_{32}-b_{22} b_{13} b_{31}-b_{33} b_{12} b_{21}, \\
A_{1}=B_{11}+B_{22}+B_{33}, \\
A_{2}=B_{11} B_{22}+B_{22} B_{33}+B_{11} B_{33}-B_{12} B_{21} \\
-B_{23} B_{32}-B_{13} B_{31}, \\
A_{3}=B_{11} B_{22} B_{33}+B_{12} B_{23} B_{31}+B_{13} B_{21} B_{32} \\
-B_{11} B_{23} B_{32}-B_{22} B_{13} B_{31}-B_{33} B_{12} B_{21}, \\
B_{i k}=\alpha^{-1} \tilde{\gamma}_{i k}, \quad b_{i k}=\alpha^{-1} \gamma_{i k} .
\end{gathered}
$$

Solving (13), we obtain eight roots of $\xi$ of the form $\pm\left(k_{j}+\right.$ $\left.i q_{j}\right), j=1,2,3,4$. Corresponding to each pair of these roots there are two waves traveling in opposite directions with distinct wave speed $v=\omega / \operatorname{Re}(\xi)$. Thus there are four distinct waves, namely, a quasi-longitudinal wave, two quasitransverse waves, and a quasi-concentration wave, which can propagate in anisotropic laser-excited solids with atomic defect generation. These waves are attenuated in space having attenuation coefficients $q_{j}, j=1,2,3,4$, which also get modified due to defect concentration variations. All the waves are coupled with each other.

In the absence of diffusion and generation-recombination effects, we obtain from (13) the polynomial characteristic equation corresponding to anisotropic elastic medium as

$$
\rho^{3} v^{6}-\rho^{2}\left(\gamma_{11}+\gamma_{22}+\gamma_{33}\right) v^{4}+\rho \alpha^{2} a_{2} v^{2}-\alpha^{3} a_{3}=0,
$$

which is similar to that obtained by Rose [29].

\section{Waves of Assigned Frequency in Transversely Isotropic Crystals}

This type of medium has only one axis of elastic symmetry that is also an axis of defect diffusion symmetry. We take $x_{3}$ axis along the axis of symmetry; then the nonvanishing elastic and atomic defect parameters are

$$
\begin{aligned}
& c_{11}=c_{22}, \quad c_{33}, c_{12}, c_{23}=c_{13}, \\
& c_{44}=c_{55}, \quad c_{66}=\frac{\left(c_{11}-c_{12}\right)}{2}, \\
& D_{11}=D_{22}=D_{1}, \quad D_{33}=D_{3}, \\
& \vartheta_{11}=\vartheta_{22}=\vartheta_{1}, \quad \vartheta_{33}=\vartheta_{3} .
\end{aligned}
$$

We will study the propagation of plane harmonic waves in different principal planes as follows.

4.1. Wave Propagation in a Principal Plane. Let us consider plane waves propagating in a principal plane perpendicular to the principal direction [010]; that is, wave normal $\vec{n}=$ $(\sin \theta, 0, \cos \theta)$ inclined at angle $\theta$ to $x_{3}$ axis. The Christoeffel stiffness is

$$
\begin{gathered}
\gamma_{11}=c_{11} \sin ^{2} \theta+c_{44} \cos ^{2} \theta, \\
\gamma_{22}=\frac{1}{2}\left(c_{11}-c_{12}\right) \sin ^{2} \theta+c_{44} \cos ^{2} \theta, \\
\gamma_{33}=c_{33} \cos ^{2} \theta+c_{44} \sin ^{2} \theta, \\
\gamma_{13}=\left(c_{33}+c_{44}\right) \sin \theta \cos \theta, \\
\gamma_{12}=\gamma_{23}=0, \\
\widetilde{\gamma}_{11}=C_{11} \sin ^{2} \theta+C_{44} \cos ^{2} \theta, \\
\tilde{\gamma}_{22}=\frac{1}{2}\left(C_{11}-C_{12}\right) \sin ^{2} \theta+C_{44} \cos ^{2} \theta, \\
\tilde{\gamma}_{33}=C_{33} \cos ^{2} \theta+C_{44} \sin ^{2} \theta, \\
\widetilde{\gamma}_{13}=\left(C_{33}+C_{44}\right) \sin \theta \cos \theta, \\
\tilde{\gamma}_{12}=\tilde{\gamma}_{23}=0,
\end{gathered}
$$


where

$$
\begin{gathered}
C_{11}=c_{11}\left[1-\frac{\left(\delta+l^{2} \xi^{2} \varepsilon\right)}{(1-i \omega \tau)}\right], \quad C_{22}=c_{44}, \\
C_{33}=c_{33}-\frac{c_{11}\left(\delta+l^{2} \xi^{2} \varepsilon\right)}{(1-i \omega \tau)}, \\
C_{12}=c_{12}-\frac{c_{11} \bar{\vartheta}\left(\delta+l^{2} \xi^{2} \varepsilon\right)}{(1-i \omega \tau)}, \\
C_{13}=c_{13}-\frac{c_{11} \bar{\vartheta}\left(\delta+l^{2} \xi^{2} \varepsilon\right)}{(1-i \omega \tau)}, \quad \bar{\vartheta}=\frac{\vartheta_{3}}{\vartheta_{1}} \\
\delta=\frac{g_{0} \beta 9^{(g m)} \vartheta_{1} \tau}{c_{11}}, \quad \varepsilon=\frac{N_{0} \beta \vartheta_{1}^{2} l^{2}}{c_{11}} .
\end{gathered}
$$

The constants $\delta$ and $\varepsilon$, defined by expressions (18b), are called the defect-strain coupling constants because they are measures of the coupling between the displacement deformation and the generation-recombination and diffusion of defects, respectively. $\delta$ and $\varepsilon$ vanish when $\vartheta_{1}$ vanishes. When $\delta$ and $\varepsilon$ are zero (2) and (6) governing the elastic waves and the defect concentration wave uncouple. An increase in deformational potentials $\left(\vartheta_{1}, \vartheta^{(g m)}\right)$ is reflected as an increase in $\delta$ and $\varepsilon$ and the coupling between defect concentration and the displacement deformation. Below we exclude the coupled parameter $\varepsilon$ from our study $(\varepsilon=0)$.

The dispersion equation (13), in this case, reduces to

$$
\begin{gathered}
\xi^{2}\left[\left(c_{11}-c_{12}\right) \sin ^{2} \theta+2 c_{44} \cos ^{2} \theta\right]-2 \rho \omega^{2}=0, \\
\sigma M\left(\eta^{2}-r_{1}\right)\left(\eta^{2}-r_{2}\right)-\eta^{2} \tau_{0}\left(\eta^{2}-r_{1}^{*}\right)\left(\eta^{2}-r_{2}^{*}\right)=0
\end{gathered}
$$

with

$$
\begin{gathered}
r_{1,2}=\frac{\left\lfloor b_{2} \pm\left(b_{2}^{2}-4 b_{1}\right)^{1 / 2}\right\rfloor}{2}, \\
r_{1,2}^{*}=\frac{\left(1+\delta / \tau_{0}\right)\left\lfloor B_{2} \pm\left(B_{2}^{2}-4 B_{1}\right)^{1 / 2}\right\rfloor}{2}, \\
b_{1}=c_{2} \sin ^{4} \theta+\left(c_{1}+c_{2}^{2}-c_{3}^{2}\right) \sin ^{2} \theta \cos ^{2} \theta+c_{1} c_{2} \cos ^{4} \theta, \\
b_{2}=\left(1+c_{2}\right) \sin ^{2} \theta+\left(c_{1}+c_{2}\right) \cos ^{2} \theta, \\
B_{1}=C_{2} \sin ^{4} \theta+\left(C_{1}+C_{2}^{2}-C_{3}^{2}\right) \sin ^{2} \theta \cos ^{2} \theta+C_{1} C_{2} \cos ^{4} \theta, \\
B_{2}=\left(1+C_{2}\right) \sin ^{2} \theta+\left(C_{1}+C_{2}\right) \cos ^{2} \theta, \\
\bar{D}=\frac{D_{3}}{D_{1}}, \quad M=\sin ^{2} \theta+\bar{D} \cos ^{2} \theta, \quad \eta^{2}=\frac{\rho \omega^{2}}{c_{11} \xi^{2}} .
\end{gathered}
$$

Equation (19) corresponds to the purely transverse (SH) wave mode, which is not affected by defect generation and is independent of $\vec{n}$. This wave propagates without dispersion or damping with speed

$$
v=\left\{\frac{\left[\left(c_{11}-c_{12}\right) \sin ^{2} \theta+2 c_{44} \cos ^{2} \theta\right]}{2 \rho}\right\}^{1 / 2}
$$

and decouples from rest of the motion.

Equation (20), being cubic in $\eta^{2}$, gives three roots and has three dispersive waves, namely, quasi-longitudinal (QL) wave, quasi-transverse (QT) wave, and concentration wave (N-mode). We can observe that these waves are affected by the anisotropy and strain-defect coupling effects.

The roots of (20), in general, can be readily solved numerically or graphically, but in some cases of interest they can also be solved analytically. We will now consider this in the following special cases.

When the strain and defect concentration fields are not coupled with each other, the defect-strain coupling constant $(\delta)$ is identically zero. Then (20) admits the following solutions:

$$
\eta_{1}^{2}=r_{1}, \quad \eta_{2}^{2}=r_{2}, \quad \eta_{3}^{2}=\frac{\sigma M}{\left(i \chi \omega^{*} \tau-1\right)}=r_{3} .
$$

Now we take into account the coupling and assume the parameter $\delta$ to be small (weak defect-elastic interaction). Therefore we develop series expansions in terms of $\delta$ for the roots $\eta_{i}^{2}(i=1,2,3)$ of $(20)$ in order to explore the effect of various interacting fields on the waves.

If the coupling term is very small $(\delta \ll 1)$ the expressions for $r_{1,2}^{*}, B_{1}$, and $B_{2}$ that appear in the dispersion equation (20), to a first approximation, then read

$$
r_{1,2}^{*}=r_{1,2}+\frac{\delta\left(d_{1,2}+r_{1,2}\right)}{\tau_{0}},
$$

$$
B_{1}=b_{1}+\frac{\delta m_{1}}{\tau_{0}}, \quad B_{2}=b_{2}+\frac{\delta m_{2}}{\tau_{0}},
$$

with

$$
\begin{gathered}
d_{1,2}=\frac{\left(-m_{2} \pm\left(2 m_{1}-m_{2} b_{2}\right) / \sqrt{b_{2}^{2}-4 b_{1}}\right)}{2} \\
m_{1}=-c_{2} \sin ^{4} \theta+\left[\bar{\vartheta}^{2}-c_{1}-2 c_{2}^{2}-2 c_{3}\left(\bar{\vartheta}-c_{3}\right)\right] \\
\cdot \sin ^{2} \theta \cos ^{2} \theta+c_{2}\left(\bar{\vartheta}^{2}-2 c_{1}\right) \cos ^{4} \theta, \\
m_{2}=-c_{2}+\left(\bar{\vartheta}^{2}-c_{1}\right) \cos ^{2} \theta .
\end{gathered}
$$

Then the secular equation (20) can be rewritten as

$$
\left(\eta^{2}-r_{1}\right)\left(\eta^{2}-r_{2}\right)\left(\eta^{2}-r_{3}\right)=\frac{\delta}{\tau_{0}} \Omega\left(\eta^{2}\right)
$$


where

$$
\Omega\left(\eta^{2}\right)=\eta^{2}\left[\left(d_{2}+r_{2}\right)\left(\eta^{2}-r_{1}\right)+\left(d_{1}+r_{1}\right)\left(\eta^{2}-r_{2}\right)\right] .
$$

Now, we may write $\eta_{i}^{2}=r_{i}+\Lambda_{i}, i=1,2,3$, where $\Lambda_{i}$ is the increment of the value of $r_{i}$ due to $\delta \neq 0$. Thus, the roots of (26) may be approximated as

$$
\begin{aligned}
& \eta_{j}^{2}=r_{j}\left[1+\frac{\delta\left(d_{j}+r_{j}\right)}{\tau_{0}\left(r_{j}-r_{3}\right)}+O\left(\delta^{2}\right)\right]=\widetilde{r}_{j}, \quad j=1,2, \\
& \eta_{3}^{2}=r_{3}\left[1+\frac{\delta}{\tau_{0}}\left(\frac{d_{1}+r_{1}}{r_{3}-r_{1}}+\frac{d_{2}+r_{2}}{r_{3}-r_{2}}\right)+O\left(\delta^{2}\right)\right]=\widetilde{r}_{3} .
\end{aligned}
$$

Upon using this representation, the corresponding phase velocities and attenuation coefficients can be obtained as

$$
\begin{gathered}
v_{i}=\frac{\left(c_{11} / \rho\right)^{1 / 2} \sqrt{z_{i}}}{\cos \left(\phi_{i} / 2\right)} \\
q_{i}=\frac{\left(c_{11} / \rho\right)^{-1 / 2} \omega \sin \left(\phi_{i} / 2\right)}{\sqrt{z_{i}}}
\end{gathered}
$$

where

$$
\begin{gathered}
z_{i}=\left(l_{i}^{2}+h_{i}^{2}\right)^{1 / 2}, \quad \phi_{i}=\arctan \left( \pm\left|\frac{h_{i}}{l_{i}}\right|\right), \\
i=1,2,3, \\
l_{i}=r_{i}\left(1-\frac{\delta\left(\sigma M+r_{i}\right)\left(d_{i}+r_{i}\right)}{s_{i}}\right), \\
h_{i}=-\delta \frac{\left(d_{i}+r_{i}\right) \chi \bar{\omega} \tau r_{i}^{2}}{s_{i}}, \\
s_{i}=\left(\sigma M+r_{i}\right)^{2}+\left(\chi \bar{\omega} \tau r_{i}\right)^{2}
\end{gathered}
$$

for elastic waves and

$$
\begin{aligned}
l_{3}= & -\frac{\sigma M}{1+(\chi \bar{\omega} \tau)^{2}} \\
& +\delta \frac{\sigma M}{1+(\chi \bar{\omega} \tau)^{2}} \sum_{i=1,2} \frac{\left(d_{i}+r_{i}\right)\left[(\chi \bar{\omega} \tau)^{2} r_{i}-\left(\sigma M+r_{i}\right)\right]}{s_{i}} \\
h_{3}= & -\frac{\sigma \chi \bar{\omega} \tau M}{1+(\chi \bar{\omega} \tau)^{2}}-\delta \frac{\sigma \chi \bar{\omega} \tau M}{1+(\chi \bar{\omega} \tau)^{2}} \sum_{i=1,2} \frac{\left(d_{i}+r_{i}\right)\left(\sigma M+2 r_{i}\right)}{s_{i}}
\end{aligned}
$$

for defect concentration waves. The "+" or "-" sign in $\phi_{i}$ is taken according to whether $x_{p} n_{p}>0$ or $<0$.

As these roots are frequency dependent, waves are dispersive character. All the waves are coupled with each other due to defect-strain interaction.
4.2. Wave Propagation in Other Planes. Let us consider propagation of plan waves in principal planes perpendicular to the principal direction [100] or [001]; that is, the normal $\vec{n}=(0, \sin \theta, \cos \theta)$ or $\vec{n}=(\sin \theta, 0, \cos \theta)$, where $\theta$ is now the inclination of wave normal to the $x_{3}$ axis and $x_{2}$ axis, respectively. The $\mathrm{SH}$ wave (purely transverse) again gets decoupled from the motion and is not affected by defect concentration variations in these directions. This wave now propagates without dispersion or damping with the velocity

$$
\begin{gathered}
v=\left\{\frac{\left[\left(c_{11}-c_{12}\right) \sin ^{2} \theta+2 c_{55} \cos ^{2} \theta\right]}{2 \rho}\right\}^{1 / 2}, \\
v=\left(\frac{c_{55}}{\rho}\right)^{1 / 2}
\end{gathered}
$$

in the principal planes perpendicular to [100] and [001], respectively.

The rest of the motion is coupled and governed by the secular equation of the type similar to (20) with minor adjustments of elastic and defect's subsystem parameters in these directions. The corresponding velocities as well as the attenuation coefficients of the constituent wave modes can be obtained by following the approach presented for Section 4.1. In all these cases the waves are found to be dispersive in character and attenuated in space.

4.3. Wave Propagation along and Perpendicular to Axis of Symmetry. For $\theta=90^{\circ}$, that is, when the wave normal $\vec{n}=$ [100] perpendicular to the axis of symmetry ( $x_{3}$ axis), the secular equation (13) reduces to three equations: two of them correspond to two purely transverse wave modes, which are not affected by change of defect concentration and are independent of $\vec{n}$. These two transverse modes travel without dispersion or damping with speeds $v=\left(c_{66} / \rho\right)^{1 / 2}$ and $v=$ $\left(c_{44} / \rho\right)^{1 / 2}$ and decouple from the rest of the motion. Third equation has the form

$$
\left(\eta^{2}-1\right)\left(\sigma M \tau_{0}^{-1}-\eta^{2}\right)=-\eta^{2} \delta \tau_{0}^{-1}
$$

In case of low frequencies, it follows from (33), for small values of $\chi$, that $\eta^{2} \rightarrow 1 /(1-\delta)$. The longitudinal elastic wave in this limiting case travels with speed $v=\left(\widetilde{c}_{11} / \rho\right)^{1 / 2}$, where $\widetilde{c}_{11}=c_{11}(1-\delta)$. For high frequencies $(\chi \gg 1)$, from (33) we can write $\eta^{2} \rightarrow 1$ and the elastic dilatation wave propagates with the classical velocity $\sqrt{c_{11} / \rho}$.

A similar type of discussion can be made for the waves propagating along the axis of symmetry, that is, for $\theta=0$.

\section{Waves of Assigned Frequency in Cubic Crystals}

A wide class of crystalline materials such as $\mathrm{Al}, \mathrm{Cu}, \mathrm{Pb}, \mathrm{Au}, \mathrm{Fe}$, $\mathrm{Ni}, \mathrm{W}, \mathrm{Si}$, and $\mathrm{Ge}$, which are some frequently used substances, belongs to crystals with cubic symmetry. The cubic crystals have nine planes of symmetry, the normals of which are on the three coordinate axes and on the coordinate planes 
making an angle $\pi / 4$ with the coordinate axes. With the chosen coordinate system along the crystalline directions, the mechanical behavior of cubic crystals can be characterized by three independent elastic constants [30].

For cubic crystals the nonvanishing elastic and atomic defect parameters are

$$
\begin{gathered}
c_{11}=c_{22}=c_{33}, \quad c_{12}=c_{23}=c_{13}, \\
c_{44}=c_{55}=c_{66}, \\
D_{i j}=D \delta_{i j}, \quad \vartheta_{i j}=\vartheta \delta_{i j}, \\
\vartheta_{i j}^{(g)}=\vartheta^{(g)} \delta_{i j}, \quad \vartheta_{i j}^{(m)}=\vartheta^{(m)} \delta_{i j} .
\end{gathered}
$$

For the plane waves propagating in a principal plane perpendicular to the principal direction [010], wave normal $\vec{n}=(\sin \theta, 0, \cos \theta)$ is inclined at angle $\theta$ to $x$-axis. The Christoeffel stiffness is

$$
\begin{gathered}
\gamma_{11}=c_{11} \sin ^{2} \theta+c_{44} \cos ^{2} \theta, \quad \gamma_{22}=c_{22}, \\
\gamma_{33}=c_{11} \cos ^{2} \theta+c_{44} \sin ^{2} \theta, \\
\gamma_{13}=\left(c_{12}+c_{44}\right) \sin \theta \cos \theta, \\
\gamma_{12}=\gamma_{23}=0, \\
\tilde{\gamma}_{11}=C_{11} \sin ^{2} \theta+C_{44} \cos ^{2} \theta, \quad \tilde{\gamma}_{22}=C_{22}, \\
\tilde{\gamma}_{33}=C_{11} \cos ^{2} \theta+C_{44} \sin ^{2} \theta, \\
\tilde{\gamma}_{13}=\left(C_{12}+C_{44}\right) \sin \theta \cos \theta, \\
\tilde{\gamma}_{12}=\tilde{\gamma}_{23}=0,
\end{gathered}
$$

where $C_{11}, C_{12}$, and $C_{22}$ are given by expressions (18a). Dispersion equation (13), in this case, leads to

$$
\begin{gathered}
\xi^{2} \tilde{\gamma}_{22}-\rho \omega^{2}=0 \\
\sigma\left(\eta^{2}-r_{1}\right)\left(\eta^{2}-r_{2}\right)-\eta^{2} \tau_{0}\left(\eta^{2}-\widetilde{r}_{1}\right)\left(\eta^{2}-\widetilde{r}_{2}\right)=0
\end{gathered}
$$

where

$$
\begin{gathered}
r_{1,2}=\frac{\left\lfloor p_{2} \pm\left(p_{2}^{2}-4 p_{1}\right)^{1 / 2}\right\rfloor}{2}, \\
\widetilde{r}_{1,2}=\frac{\left(1+\delta / \tau_{0}\right)\left\lfloor P_{2} \pm\left(P_{2}^{2}-4 P_{1}\right)^{1 / 2}\right\rfloor}{2}, \\
p_{1}=c_{1}+\left(1+c_{1}^{2}-2 c_{1}-c_{2}^{2}\right) \sin ^{2} \theta \cos ^{2} \theta, \\
p_{2}=1+c_{1}, \\
P_{1}=C_{1}+\left(1+C_{1}^{2}-2 C_{1}-C_{2}^{2}\right) \sin ^{2} \theta \cos ^{2} \theta, \\
P_{2}=1+C_{1},
\end{gathered}
$$

$$
\begin{gathered}
C_{1}=\frac{c_{1}}{1+\delta / \tau_{0}}, \quad C_{2}=\frac{c_{2}+\delta / \tau_{0}}{1+\delta / \tau_{0}}, \\
c_{1}=\frac{c_{44}}{c_{11}}, \quad c_{2}=\frac{\left(c_{12}+c_{44}\right)}{c_{11}} .
\end{gathered}
$$

Equation (36) gives us a pair of roots $\xi= \pm \omega\left(\rho / c_{44}\right)^{1 / 2}$. This pair corresponds to purely transverse ( $\mathrm{SH})$ wave mode, which is not affected by defect generation and is independent of $\vec{n}$. This wave propagates without dispersion or damping with speed $v=\left(c_{44} / \rho\right)^{1 / 2}$. Equation (37) is similar to (20) and gives three roots and has three dispersive waves, namely, quasi-longitudinal (QL) wave, quasi-transverse (QT) wave, and defect concentration wave (N-mode), depending on the defect relaxation time $(\tau)$ and strain-defect coupling effects $(\delta)$. The roots of (37) can be solved analytically by following the approach presented in Section 4.

\section{Isotropic Solids}

For isotropic solids every direction is a direction of elastic as well as diffusion symmetry and the nonvanishing elastic and defect parameters are

$$
\begin{gathered}
c_{11}=c_{22}=c_{33}=\lambda+2 \mu, \\
c_{12}=c_{23}=c_{13}=\lambda, \\
c_{44}=c_{55}=c_{66}=\mu, \\
D_{1}=D_{2}=D_{3}=D, \\
\vartheta_{i j}=9 \delta_{i j}, \quad \vartheta_{i j}^{(g)}=\vartheta^{(g)} \delta_{i j}, \\
\vartheta_{i j}^{(m)}=\vartheta^{(m)} \delta_{i j} .
\end{gathered}
$$

Using these values of parameters in the foregoing analysis we obtain the results of plane harmonic wave propagation in isotropic solids [31].

\section{Numerical Results}

To study in detail the dependence of velocities and attenuation coefficient of plane waves on their direction of propagation, we consider single crystal of $\mathrm{Zn}$ as transversely isotropic elastic solid for which the basic physical data are given in Table 1 .

The numerical values of the velocities and attenuation coefficients of waves have been calculated by using (29) for different values of the angle of incidence modified in the range $0 \leq \theta \leq \pi / 2$. In Figures $1-6$ we present the generation-diffusion effects in a defect subsystem on variations of nondimensional phase velocities $V_{j}=v_{j} / v_{1}\left(v_{1}=\right.$ $\sqrt{c_{11} / \rho}$ is the longitudinal wave velocity in the medium) and nondimensional attenuation coefficients $Q_{j}=q_{j} / q_{0}, q_{0}=$ $10^{7} \mathrm{~m}^{-1}$, for various directions of propagation with respect to nondimensional frequency $\chi$ (on log-linear scales). 
TABLE 1: Basic data for single crystal of zinc.

\begin{tabular}{lccccccc}
\hline Const. & $c_{11}$ & $c_{13}$ & $c_{44}$ & $c_{33}$ & $\rho$ & $\delta$ & $D$ \\
\hline Unit & $\mathrm{Nm}^{-2}$ & $\mathrm{Nm}^{-2}$ & $\mathrm{Nm}^{-2}$ & $\mathrm{Nm}^{-2}$ & $\mathrm{Kgm}^{-3}$ & - & $\mathrm{m}^{2} \mathrm{c}^{-1}$ \\
\hline Zn & $1.628 \times 10^{11}$ & $0.508 \times 10^{11}$ & $0.385 \times 10^{11}$ & $0.627 \times 10^{11}$ & 7140 & 0.1 & $4 \cdot 10^{-7}$ \\
\hline
\end{tabular}

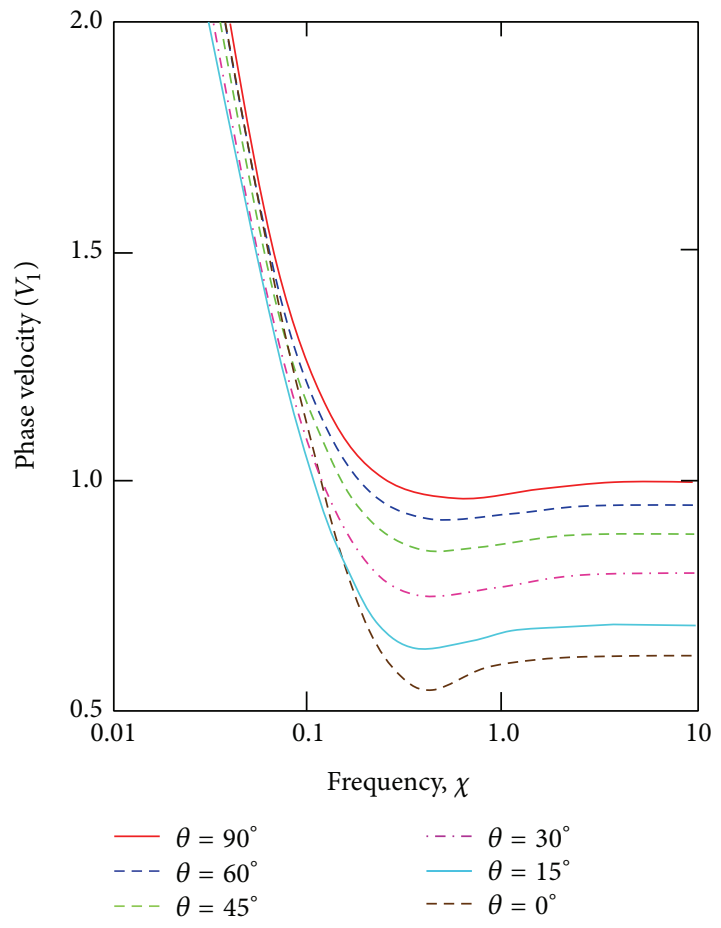

FIGURE 1: Variation of QL wave velocity with nondimensional frequency in various direction of propagation.

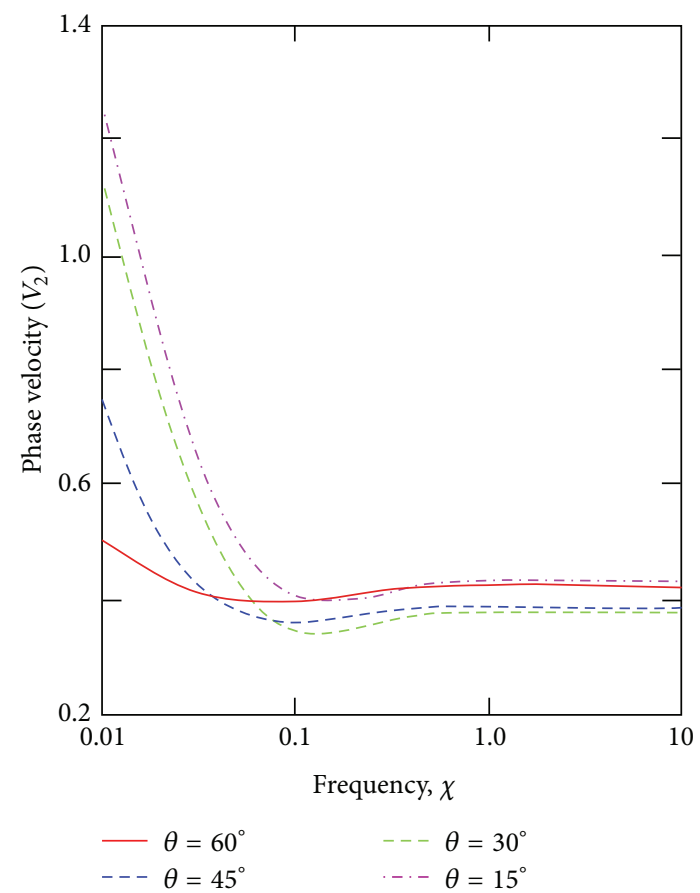

FIGURE 2: Variation of QT wave velocity with nondimensional frequency in various direction of propagation.

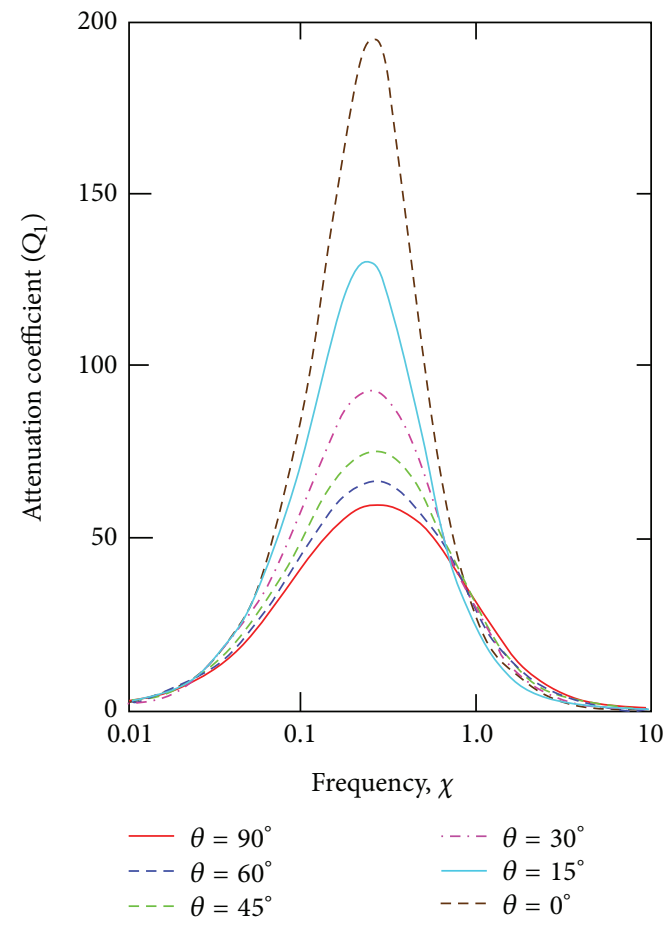

FIGURE 3: Variation of QL attenuation coefficient with nondimensional frequency in various direction of propagation.

From the curves in Figure 1 it is observed that for each value of $\theta$ the variations of phase velocity of QL waves with frequency decrease for $\chi=0.01$ to $\chi=0.4$, increase for $0.4 \leq \chi \leq 1.0$, and then remain almost constant. The QL waves propagating in the direction inclined at an angle of $\theta=0^{\circ}$ with the axis of symmetry are less affected due to the defect concentration variations than those propagating in other directions.

The phase velocity of modified QT waves (Figure 2) first decreases for $\chi=0.01$ to $\chi=0.2$ and then increases for $0.2 \leq \chi \leq 0.5$ and remains constant for $\chi \rightarrow \infty$. It is observed from Figure 2 that the variations of phase velocity of QT waves are lower in magnitude as compared to those in Figure 1. The velocity of QT waves propagating in directions $\theta=60^{\circ}, 75^{\circ}$ varies slightly. It justifies the fact that shear wave is less affected due to defect-strain interactions.

Figures 3 and 4 show the behaviors of attenuation coefficients of the modified QL and QT waves with respect to $\chi$, respectively. We note that the values of the attenuation coefficients of the QL waves are positive and increase for $0.01<\chi<0.3$ and decrease monotonically for $\chi>$ 0.3 and ultimately become close to zero as $\chi \rightarrow \infty$. Figure 4 indicates that the trend of variations of attenuation 


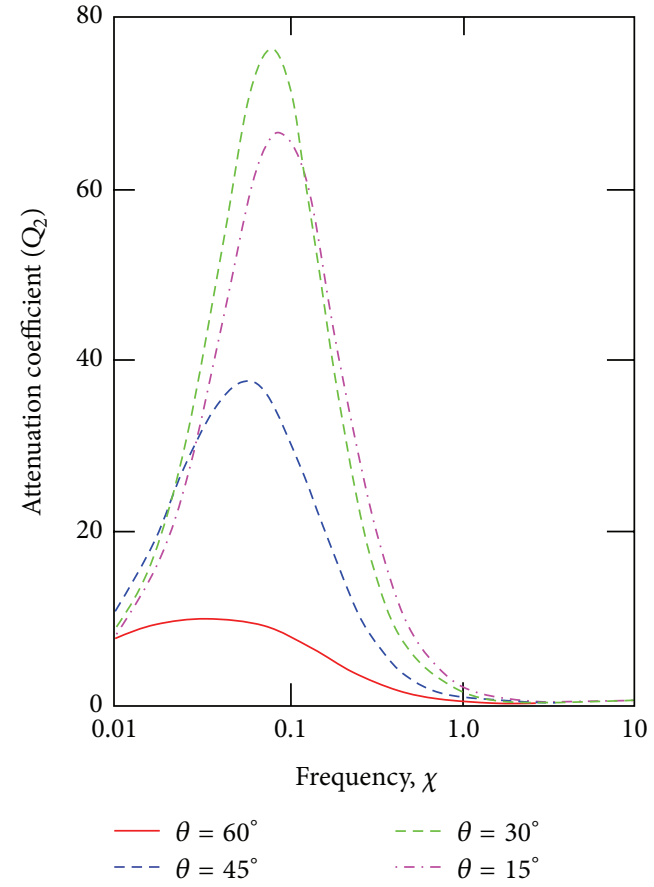

FIGURE 4: Variation of QT attenuation coefficient with nondimensional frequency in various direction of propagation.

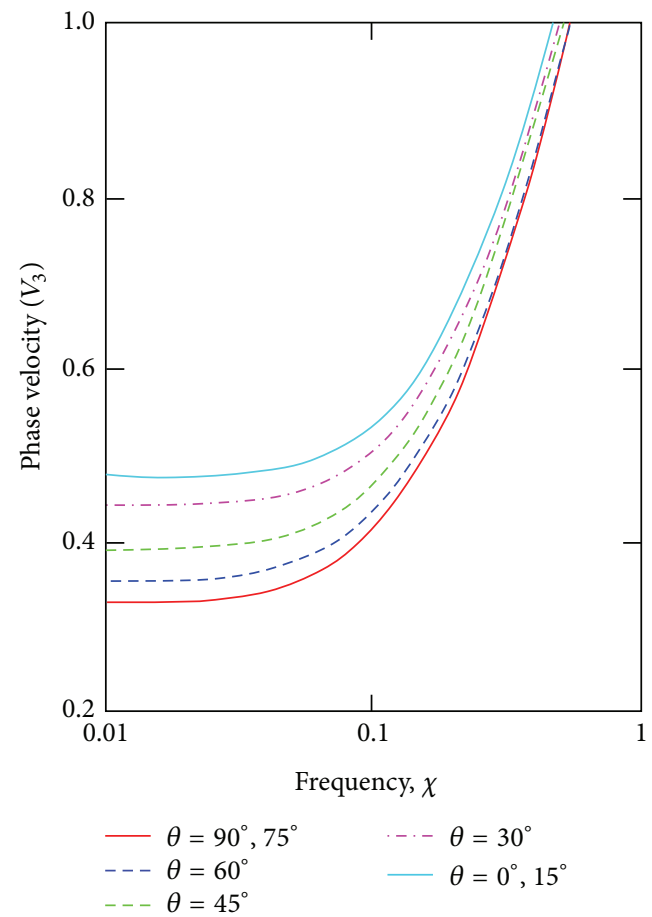

FIGURE 5: Variation of phase velocity of the N-mode with nondimensional frequency in various direction of propagation.

coefficients of QT waves is similar to that of QL waves, but no modifications are observed for directions $\theta=60^{\circ}, 75^{\circ}$.

The curves in Figures 5 and 6 show that the phase velocity and the attenuation coefficient of defect concentration wave

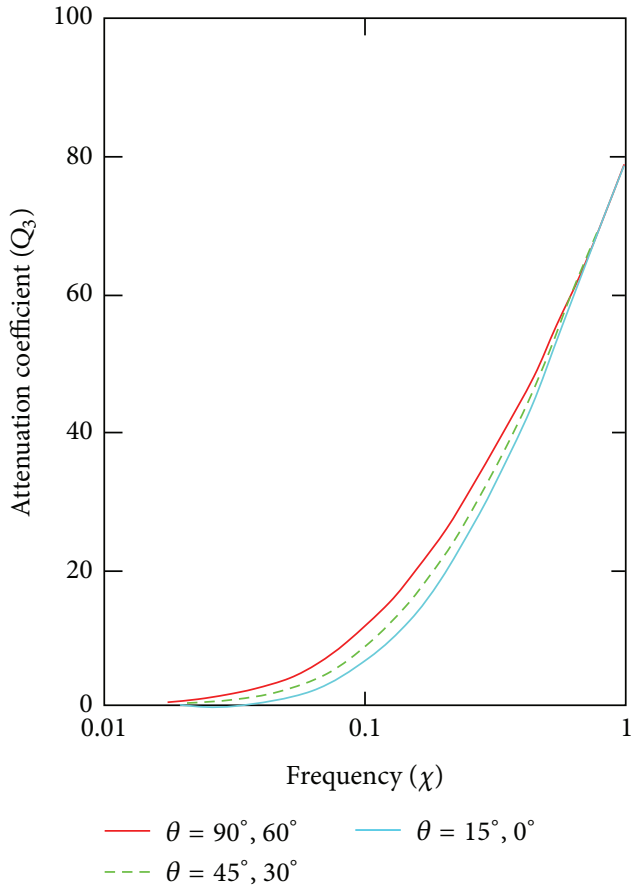

FIGURE 6: Variation of attenuation coefficient of the N-mode with nondimensional frequency in various direction of propagation.

increase monotonically for all values of $\chi$. These curves suggest that approximately there is no effect of anisotropy of the medium on the attenuation coefficients of $\mathrm{N}$-mode. Some changes of the phase velocity of $\mathrm{N}$-mode for various direction of propagation can be observed for small values of frequency. No modifications are noticed due to anisotropy on velocity of these waves for high frequencies.

\section{Conclusions}

In the present paper the combined effect of anisotropy and atomic defect generation on the dispersive wave propagation in solids subjected to action of laser pulses has been studied, in the context of the coupled model based on equations for the displacement vector of the medium and defect dynamics. The dispersion relation solutions of quasi-plane waves have been derived. According to the dispersion equations four types of waves, a quasi-longitudinal wave (QL-mode), two quasi-transverse waves (QT-mode), and a quasi-defect concentration wave (N-mode), can, in general, propagate in considered crystals. The analytical expressions of phase velocity and attenuation coefficients have been obtained. Some special cases of interest have been deduced from the present investigation. It may be concluded that the anisotropic and defect concentration changes play an important role on the wave propagation in laser-excited solids.

It is demonstrated that in case of wave propagation in one of the planes of transversely isotropic elastic solid having defect concentration field only one purely quasi-transverse (QT) wave decouples from the rest of the motion and is not affected by the defect concentration changes. The other waves 
are coupled and get modified due to presence of defects. On other hand, when plane waves propagate along the axis of the solid, then only longitudinal and defect concentration waves are coupled, whereas the two quasi-transverse (QT) wave modes get decoupled from the rest of the motion.

From analytical and graphical results for the single crystal of $\mathrm{Zn}$ it is clear that the effects of the anisotropy and the defect-strain coupling parameter are significant on the behaviors of phase velocity and attenuation coefficients with respect to frequency. The maximum variations in the velocities occur in the low frequency range, whereas these quantities for QL- and QT-modes almost remain constant in the high frequency range. Also it has been observed that there is an appreciable variation in case of QL-mode as compared with QT and defect concentration modes. Some particular cases are also considered.

\section{Conflict of Interests}

The author declares that there is no conflict of interests regarding the publication of this paper.

\section{Acknowledgments}

The author wishes to thank reviewers for their valuable suggestions for the improvement of the paper in its present form. The work was supported by the Federal Purpose Program "Research and development on priority directions of development of scientific-technological complex of Russia for 20142020" Contract no. 14.626.21.0001 from 03 October 2014.

\section{References}

[1] F. Kh. Mirzade, "Concentration-elastic instabilities in a solid half-space," Physica Status Solidi B: Basic Research, vol. 246, no. 7, pp. 1597-1603, 2009.

[2] F. Kh. Mirzade, "On diffusion-elastic instabilities in a solid halfspace," Physica B: Condensed Matter, vol. 406, no. 1, pp. 119-124, 2011.

[3] F. Kh. Mirzade, "Size effects on surface elastic waves in a semiinfinite medium with atomic defect generation," Advances in Condensed Matter Physics, vol. 2013, Article ID 528208, 11 pages, 2013.

[4] F. Kh. Mirzade, "Small-scale effect on longitudinal wave propagation in laser-excited plates," Journal of Nanoscience, vol. 2014, Article ID 513010, 8 pages, 2014.

[5] D. Walgraef, N. M. Ghoniem, and J. Lauzeral, "Deformation patterns in thin films under uniform laser irradiation," Physical Review B, vol. 56, no. 23, Article ID 15361, 7 pages, 1997.

[6] D. Walgraef and N. M. Ghoniem, "Modeling laser-induced deformation patterns: nonlinear effects and numerical analysis," Journal of Computer-Aided Materials Design, vol. 6, no. 2, pp. 323-335, 1999.

[7] F. Kh. Mirzade, "Nonlinear strain waves interacting with laser induced carries of the local disorder," in Laser Technologies of Materials Treatment, V. Y. Panchenko, Ed., pp. 220-277, Fizmatlit, Moscow, Russia, 2009, (Russian).

[8] F. Kh. Mirzade, "Finite-amplitude strain waves in laser-excited plates," Journal of Physics Condensed Matter, vol. 20, no. 27, Article ID 275202, 2008.
[9] F. Kh. Mirzade, "A model for the propagation of strain solitary waves in solids with relaxing atomic defects," Journal of Applied Physics, vol. 103, no. 4, Article ID 044904, 2008.

[10] S. Bargmann and A. Favata, "Continuum mechanical modeling of laser-pulsed heating in polycrystals: a multi-physics problem of coupling diffusion, mechanics, and thermal waves," Zeitschrift für Angewandte Mathematik und Mechanik, vol. 94, no. 6, pp. 487-498, 2014.

[11] P. Steinmann, A. T. McBride, S. Bargmann, and A. Javili, "A deformational and configurational framework for geometrically non-linear continuum thermomechanics coupled to diffusion," International Journal of Non-Linear Mechanics, vol. 47, no. 2, pp. 215-227, 2012.

[12] A. T. McBride, A. Javili, P. Steinmann, and S. Bargmann, "Geometrically nonlinear continuum thermomechanics with surface energies coupled to diffusion," Journal of the Mechanics and Physics of Solids, vol. 59, no. 10, pp. 2116-2133, 2011.

[13] T. Mura, Micromechanics of Defects in Solids, Martinus Nijhoff Publishers, Dordrecht, The Netherlands, 1987.

[14] S. Y. Hu and L. Q. Chen, "Solute segregation and coherent nucleation and growth near a dislocation-a phase-field model integrating defect and phase microstructures," Acta Materialia, vol. 49, no. 3, pp. 463-472, 2001.

[15] S. Y. Hu and L. Q. Chen, "Diffuse-interface modeling of composition evolution in the presence of structural defects," Computational Materials Science, vol. 23, no. 1-4, pp. 270-282, 2002.

[16] Y. Wang and J. Li, "Phase field modeling of defects and deformation," Acta Materialia, vol. 58, no. 4, pp. 1212-1235, 2010.

[17] M. J. P. Musgrave, Crystal Acoustics, Holden-Day, San Francisco, Calif, USA, 1970.

[18] E. Dieulesaint and D. Royer, Elastic Waves in Solids, John Wiley \& Sons, New York, NY, USA, 1980.

[19] P. Chadwick and L. T. Seet, "Wave propagation in a transversely isotropic heat-conducting elastic material," Mathematika, vol. 17, pp. 255-274, 1970.

[20] P. Chadwick, "Basic properties of plane harmonic waves in a prestressed heat-conducting elastic material," Journal of Thermal Stresses, vol. 2, no. 2, pp. 193-214, 1979.

[21] R. Kumar and T. Kansal, "Propagation of Lamb waves in transversely isotropic thermoelastic diffusive plate," International Journal of Solids and Structures, vol. 45, no. 22-23, pp. 58905913, 2008.

[22] R. Kumar and R. Kumar, "Propagation of plane waves in an anisotropic thermoelastic material with voids under initial stress," International Journal of Applied Mathematics and Mechanics, vol. 8, no. 17, pp. 76-97, 2012.

[23] S. Sharma, K. Sharma, and R. R. Bhargava, "Effect of viscosity on wave propagation in anisotropic thermoelastic with GreenNaghdi theory type-II and type-III," Materials Physics and Mechanics, vol. 16, no. 2, pp. 144-158, 2013.

[24] R. Kumar and R. R. Gupta, "Propagation of waves in transversely isotropic micropolar generalized thermoelastic half space," International Communications in Heat and Mass Transfer, vol. 37, no. 10, pp. 1452-1458, 2010.

[25] S. H. Guo, "The eigen theory of waves in piezoelectric solids," in Acoustic Waves, D. Dissanayake, Ed., Sciyo, Rijeka, Croatia, 2010.

[26] J. N. Sharma and M. Pal, "Propagation of lamb waves in a transversely isotropic piezothermoelastic plate," Journal of Sound and Vibration, vol. 270, no. 4-5, pp. 587-610, 2004. 
[27] D. P. Acharya, I. Roy, and S. Sengupta, "Effect of magnetic field and initial stress on the propagation of interface waves in transversely isotropic perfectly conducting media," Acta Mechanica, vol. 202, no. 1-4, pp. 35-45, 2009.

[28] A. M. Kosevich, Physical Mechanics of Real Crystals, Nauka, Moscow, Russia, 1986.

[29] J. L. Rose, Ultrasonic Waves in Solid Media, Cambridge University Press, Cambridge, UK, 1999.

[30] C. Kittel, Introduction to Solid State Physics, John Wiley \& Sons, New York, NY, USA, 1996.

[31] F. Kh. Mirzade, "Elastic wave propagation in a solid layer with laser-induced point defects," Journal of Applied Physics, vol. 110, no. 6, Article ID 064906, 2011. 

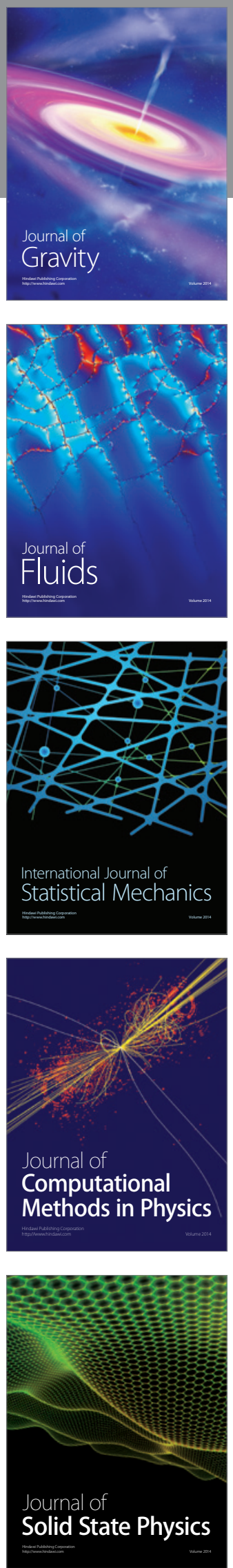

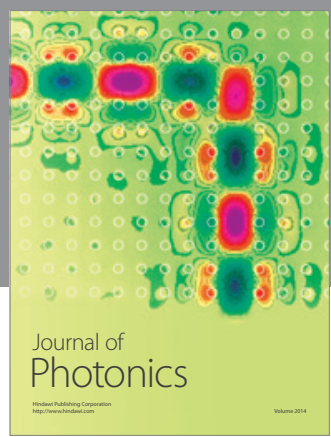

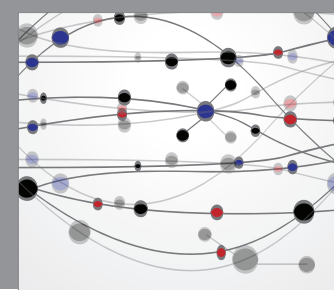

The Scientific World Journal

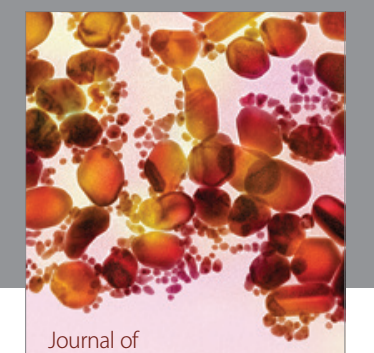

Soft Matter
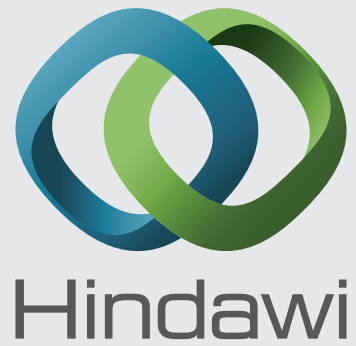

Submit your manuscripts at

http://www.hindawi.com
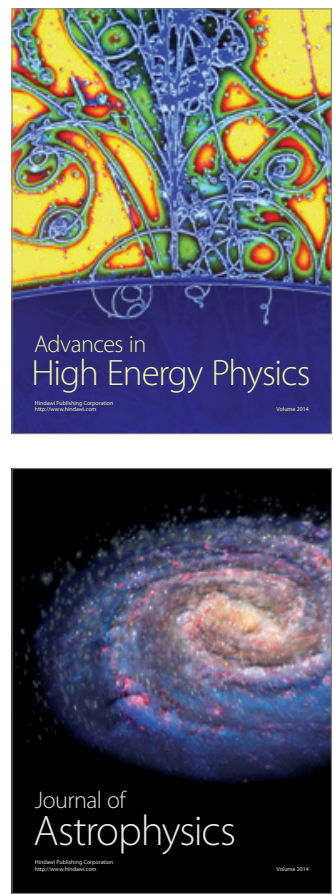
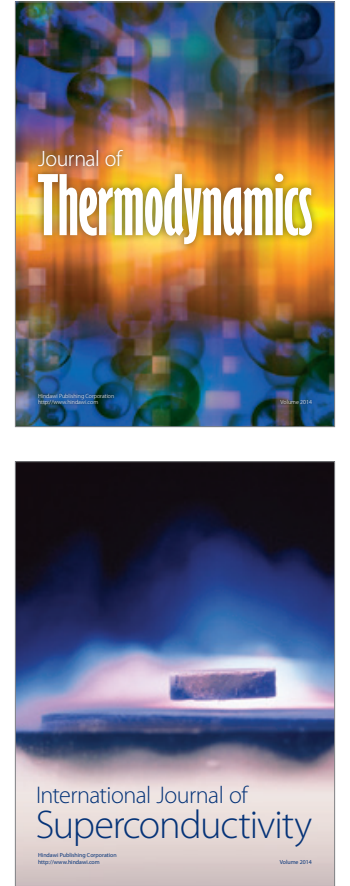
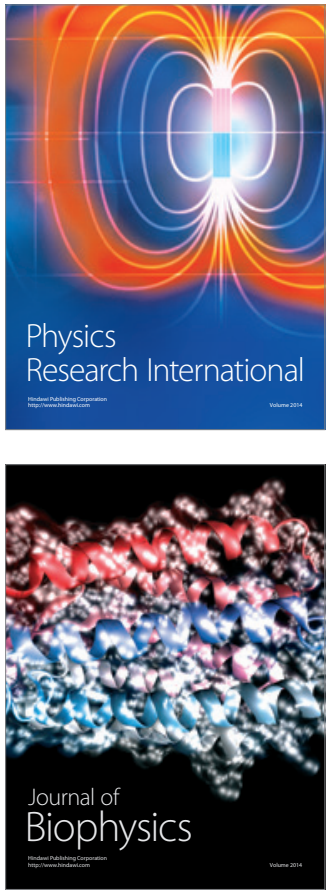
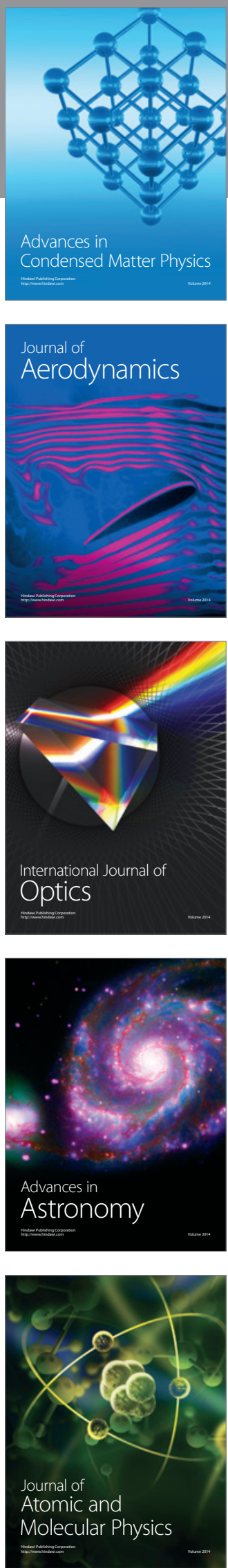